\title{
Use of Cholecystokinin to Prevent the Development of Parenteral Nutrition-Associated Cholestasis*
}

\author{
Daniel H. Teitelbaum, MD $\dagger$; Theresa Han-Markey, MS, RD $\dagger$; Robert A. Drongowski, MS $\dagger$; Arnold G. Coran, MD $\dagger$; \\ Bilge Bayar, BS†; James D. Geiger, MD†; Neal Uitulugt, MD†; And M. Anthony Schork, PhD \\ From the $\div$ Department of Surgery, Section of Pediatric Surgery, and $\doteqdot$ The School of Public Health, University of Michigan Medical Center and \\ the C. S. Mott Children's Hospital, Ann Arbor, Michigan
}

\begin{abstract}
Background: Neonates are at high risk for the development of parenteral nutrition-associated cholestasis when receiving a prolonged course of total parenteral nutrition (TPN). Although this cholestasis is of unknown etiology, it may result from a lack of gastrointestinal hormone formation, including cholecystokinin, which normally occurs after enteral feedings. Methods: Two groups of neonates were studied. The treatment group consisted of 21 consecutive, prospectively enlisted neonates receiving TPN for $>14$ days. The nontreatment group consisted of 21 infants from the 2 years preceding the study who were matched to the treatment group by gestational age, diagnosis, and duration of TPN. The major outcome determinant was direct bilirubin. Cholestasis was defined as a direct bilirubin $>2.0$ $\mathrm{mg} / \mathrm{dL}$ and was considered severe if the direct bilirubin was $>5.0$
\end{abstract}

$\mathrm{mg} / \mathrm{dL}$ after other causes were ruled out. Results: The mean direct bilirubin levels in the nontreated group progressively rose over time, whereas the mean direct bilirubin in the treated group remained level. The incidence of infants with a direct bilirubin $>2.0 \mathrm{mg} / \mathrm{dL}$ was $24 \%$ and $43 \%$ in the CCK + and CCK - groups, respectively, and was not significant $(p=.14)$. The percentage of infants with a direct bilirubin $>5.0 \mathrm{mg} / \mathrm{dL}$ was $9.5 \%$ and $38 \%$ in the treatment and nontreatment groups, respectively, and was significant, $p=.015$. Conclusions: Levels of direct bilirubin were lower in the treated compared with the nontreated group. These findings suggest that cholecystokinin prophylaxis in high-risk neonates may help prevent the development of parenteral nutrition-associated cholestasis. (Journal of Parenteral and Enteral Nutrition 21:100-103, 1997)
Parenteral nutrition-associated cholestasis (PNAC) is a common problem encountered in neonates who cannot receive adequate amounts of enteral nutrition for prolonged periods of time. ${ }^{1-3}$ The etiology of this disorder remains unclear. One possible explanation, however, is that a lack of enteral feeding leads to diminished bile flow. Because cholecystokinin (CCK) has been demonstrated to increase both intrahepatic and extrahepatic bile flow, ${ }^{4-6}$ we hypothesized that CCK may prevent the development of PNAC. Our group and others have recently reported that CCK can decrease the level of hyperbilirubinemia in neonates who have already developed PNAC., 7 The aim of this study was to determine if prophylactically administered CCK would reduce the severity of PNAC in neonates at risk for the development of this process.

\section{MATERIALS AND METHODS}

Two groups of infants receiving total parenteral nutrition (TPN) were studied. The treatment group $(\mathrm{CCK}+)$ consisted of 26 neonates who were consecutively enrolled

*Presented at the American Pediatric Surgical Association Meeting, San Diego, CA, May, 1996.

Received for publication, August 5, 1996. Accepted for publication, October 28, 1996.

Correspondence and reprint requests: Daniel H. Teitelbaum, MD, Section of Pediatric Surgery, University of Michigan Hospitals, C. S. Mott Children's Hospital, F3970, Box 0245, Ann Arbor, Michigan 48109. and followed in a prospective fashion. All patients had a disease process (eg, prematurity $[<1500 \mathrm{~g}]$, gastroschisis, necrotizing enterocolitis, or congenital diaphragmatic hernia) in which it was thought that the patient would require at least 2 weeks of TPN. A synthetic form of cholecystokinin-octapeptide (Sincalide; Bracco Diagnostics, Princeton, $\mathrm{NJ}$, referred to as CCK for the remainder of this paper) was given at a dose of $0.02 \mu \mathrm{g} / \mathrm{kg} / \mathrm{dose} I \mathrm{~V}$, twice a day for 14 days. Dosing was increased to $0.04 \mu \mathrm{g} / \mathrm{kg} /$ dose IV three times a day if TPN was continued for $>14$ days. CCK administration was terminated when $50 \%$ or more of the infants nutritional intake was received by the enteral route or upon discharge from the neonatal intensive care unit. Infants were excluded from the study if they had liver dysfunction or hyperbilirubinemia from other causes (eg, congenital obstruction of the biliary tree, hemolysis, or if they were receiving a course of extracorporeal membrane oxygenation, in which hemolysis may occur). Infants were also excluded if they had (or were suspected of having) a metabolic defect or if they had been receiving TPN for $>10$ days prior to starting on the study. Infants were removed from the study if they achieved $>50 \%$ of their caloric intake by the enteral route before completing at least 14 days of the study.

The nontreatment group (CCK-), consisted of 21 neonates who were matched to the study patients by gestational age (within 1 week), principal clinical diagnosis and duration of TPN (within 1 week). All control neonates had been treated in the same neonatal intensive care unit over the previous 2 years. All patients received the same type of parenteral nutrition. The crystalline amino acid was 
Aminosyn (Abbott Laboratories, Abbott Park, IL) and the lipid was Liposyn 20\% (Abbott Laboratories).

The primary outcome measure was direct bilirubin. PNAC was defined, based on previously established definitions, as a direct bilirubin $>2.0 \mathrm{mg} / \mathrm{dL}$ after a prolonged course of TPN (ie, $>2$ weeks) and when other causes were ruled out. ${ }^{9}$ Severe PNAC was defined as a direct bilirubin $>5.0 \mathrm{mg} / \mathrm{dL}$. Severe PNAC was defined by previous reports, which have demonstrated a high degree of mortality associated with a direct bilirubin in this range.$^{10}$ Direct bilirubin levels were obtained on a weekly basis while all infants were on the study for both study groups. Thus, bilirubin levels were obtained at similar time periods and intervals in each group, which allowed for a ready comparison. Other etiologies of hyperbilirubinemia were evaluated by clinical inspection, ultrasonography of the hepatobiliary tree, as well as hepatitis and TORCH titers. An open liver biopsy was done in three patients after the termination of the study in conjunction with another operative procedure (closure of ostomies). A cholestatic histologic appearance was present in all cases, consistent with PNAC.

A data acquisition sheet was maintained on each patient. Direct, indirect, and total bilirubins, alkaline phosphatase, alanine transaminase (ALT), asparatate transaminase (AST), and lactate dehydrogenase (LDH) were measured on a weekly basis, starting at the initiation of the study and ending at the completion of the study. Monitoring also included the amount and type of parenteral and enteral nutrition. Mean total calories and amounts of protein and lipid were calculated weekly and expressed as the mean amount delivered each day. Additionally, clinical diagnosis, birth weight, gestational age, daily body weights, operative procedures, complications, and drugs administered were recorded. Adverse reactions to CCK were monitored on a daily basis.

This study was approved by the University of Michigan's Investigational Review Board as well as by the Food and Drug Administration, which considered Sincalide an investigational drug in the neonatal period. Informed consent was obtained from the infant's parent(s) or guardian(s) prior to beginning the study.

Results are expressed as means \pm SD. The McNemar's test, linear regression analysis, and Fisher's Exact Test were used for statistical analysis; a $p$ value $<.05$ was considered significant.

\section{RESULTS}

Twenty-six neonates were recruited into the treatment group $(\mathrm{CCK}+)$. Five infants were removed from the study because they achieved $>50 \%$ of nutritional intake by the enteral route before receiving 14 days of CCK. Twentyone neonates completed the study. CCK treatment was discontinued for one patient in the treatment group at 21 days of treatment because of a persistent delay in gastric emptying that was thought to be due to the drug. Following cessation of the drug, no improvement in feeding tolerance or gastric emptying was noted. Patients in the CCK + group received a mean of $34 \pm 33$ days of CCK treatment (range 14 to 116 days). Table I shows the demographic characteristics of the two groups.

The diagnoses of the patients in each study group were as follows: gastroschisis $(n=5)$; necrotizing enterocolitis
TABLE I

Demographic data in the cholecystokinin-treated es -nontreated groups

\begin{tabular}{lccc}
\hline Variable & Treated & Nontreated & $p$ value \\
\hline Gestational age (weeks) & $31.8 \pm 5.5$ & $32.7 \pm 4.9$ & .588 \\
Age at start of study (weeks) & $1.61 \pm .98$ & $1.28 \pm 3.7$ & .467 \\
Birth weight (kg) & $1.64 \pm 1.0$ & $1.92 \pm .93$ & .350 \\
Birth weight percentile & $9.9 \pm 20.2$ & $9.4 \pm 16.4$ & .934 \\
Apgar at 1 minute & $4.50 \pm 2.9$ & $5.16 \pm 2.5$ & .480 \\
Apgar at 5 minute & $7.13 \pm 1.7$ & $7.37 \pm 1.8$ & .703 \\
& & & \\
Variable & Treated & Nontreated & $p$ value \\
Sex & & & \\
$\quad$ Male & $7(33.3)$ & $8(38.1)$ & .747 \\
$\quad$ Female & $14(66.7)$ & $13(61.9)$ & \\
Surgery & & & \\
$\quad$ Yes & $15(71.4)$ & $14(66.6)$ & .920 \\
$\quad$ No & $6(28.6)$ & $7(33.3)$ & \\
Status & & & \\
$\quad$ Lived & $18(85.7)$ & $18(85.7)$ & 1.00 \\
$\quad$ Died & $3(14.3)$ & $3(14.3)$ & \\
\hline
\end{tabular}

Values are number of subjects with percentages in parentheses.

( $n=10)$; congenital diaphragmatic hernia $(n=3)$; and lowbirth-weight infants $(n=3)$. As can be seen, the majority of the neonates had an abnormality of the gastrointestinal tract that prevented them from taking enteral feedings. Of the 10 infants in each group who were diagnosed with necrotizing enterocolitis (NEC), 6 were treated conservatively with antibiotics and nasogastric decompression and 4 underwent a surgical exploration with resection of involved intestine.

Three patients died in each group, from either their primary disease process, sepsis, or liver failure secondary to the cholestasis. All deaths occurred $>2$ weeks after the termination of the study. Two patients in both the CCK + and the CCK - groups developed a septic episode, defined as a blood culture positive for a bacteria, during the course of the study.

Mean total calories (enteral and parenteral combined) and protein and fat delivery (from parenteral nutrition) were not statistically different $(p>.05)$ between the two groups except for a higher protein delivery in the CCK+ group during the second week of the study.

Bilirubin levels are shown in Figure 1. Because of a limited number of patients remaining on the study after 7 weeks, only the first 7 weeks of the study are shown in Figure 1. Mean direct bilirubin showed a more rapid rise in the CCK - group as the period of time patients received TPN increased, whereas the mean levels of direct bilirubin were almost flat in the $\mathrm{CCK}+$ group. Although these results were not statistically different, the use of a linear regression analysis (the lowest $p=.15$ ) revealed a trend. The percentage of infants with a direct bilirubin $>2.0 \mathrm{mg} /$ dL was $24 \%$ and $43 \%$ in the CCK + and CCK- groups, respectively, and was not significant $(p=.14)$. The percentage of infants with a direct bilirubin $>5.0 \mathrm{mg} / \mathrm{dL}$ (severe cholestasis) was $9.5 \%$ and $38 \%$ in the $\mathrm{CCK}+$ and $\mathrm{CCK}-$ groups, respectively, which was significant $(p=.015)$. With longer durations of TPN, total bilirubin levels were also noted to be higher in the CCK - group compared with the CCK + group. Indirect bilirubin levels, although somewhat higher in the $\mathrm{CCK}$ - group for the first 3 weeks of the study, showed no difference between groups for other study time points. 


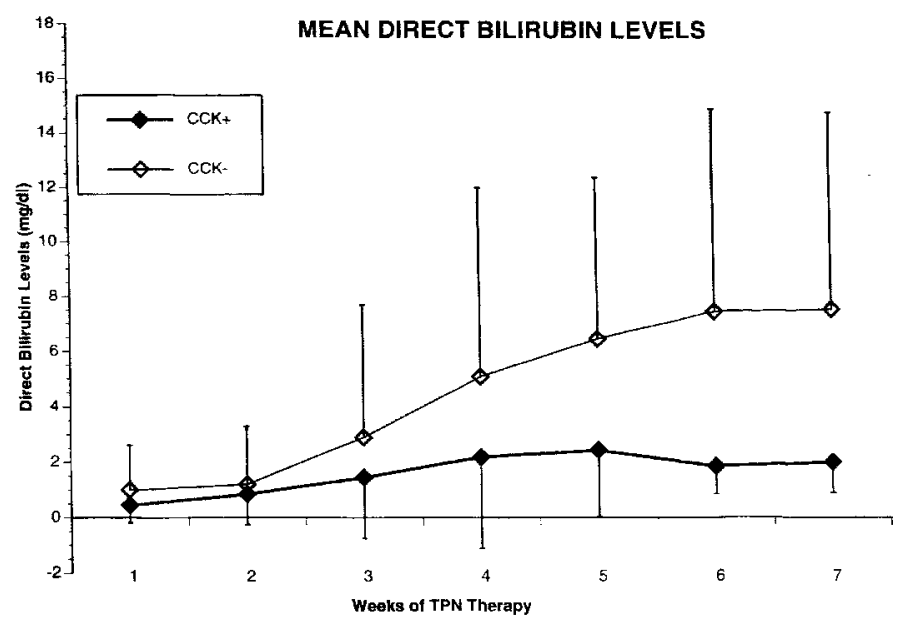

FIG. 1. Serum direct bilirubin levels measured weekly, in both groups of neonates receiving total parenteral nutrition (TPN) therapy $\left({ }^{*} p<.05\right.$ ). After the third week, note the rapid rise and consistently higher levels for the nontreatment group of patients. Because of a loss of patients in each group after week 7 , only weeks 1 through 7 are shown.

No statistical differences were noted for AST, ALT, LDH, and alkaline phosphatase levels throughout the study period.

No difference was seen in the growth characteristics of either group as measured by weight gain throughout the study. At study week 3 , body weights were $1.91 \pm 1.1$ and $2.06 \pm 1.1 \mathrm{~kg}$ in the CCK + and CCK - groups, respectively. Mean serum total protein levels, measured on a weekly basis, were virtually the same in the two groups. At study week 3 , total protein levels were $4.6 \pm 0.9$ and $4.6 \pm 1.2 \mathrm{~g} /$ $\mathrm{dL}$ in the CCK + and CCK - groups, respectively.

\section{DISCUSSION}

Parenteral nutrition-associated cholestasis (PNAC) is a persistent problem in newborns receiving long-term parenteral nutrition. ${ }^{2,11}$ The etiology of PNAC has not been identified; however, one hypothesis is that a lack of oral feedings may lead to biliary stasis. ${ }^{12}$ Biliary stasis has been observed in patients receiving prolonged courses of TPN using ultrasonographic assessment of the gallbladder. ${ }^{13}$ This stasis may develop secondarily to inadequate hormonal stimulation, which is needed for gallbladder contraction and intrahepatic bile flow. The gastrointestinal hormone cholecystokinin, which is normally released during enteral feeding and which is needed for bile flow, remains at very low levels during periods of fasting. ${ }^{14}$ Cholecystokinin has the ability to induce gallbladder contraction as well as to increase intrahepatic bile flow. ${ }^{4-6}$ Therefore, we hypothesized that cholecystokinin would be efficacious in the prevention of parenteral nutrition-associated cholestasis.

In our study, we showed that the CCK-treated neonates maintained a lower level of direct bilirubins throughout the duration of the study. The nontreated group, on the other hand, showed a steady rise in direct bilirubin levels, demonstrating a definite trend over the duration of their
TPN course. A statistically significant difference was achieved in the ability of CCK to reduce the incidence severe cholestasis (direct bilirubin $>5.0 \mathrm{mg} / \mathrm{dL}$ ); howeve significance was not attained when examining all cases PNAC (defined as a direct bilirubin $>2.0 \mathrm{mg} / \mathrm{dL}$ ). The diftficulty in achieving a significant difference was due to the fairly large standard deviations in mean direct bilirubin levels in the control group. Further, because most neonates had started on enteral feedings after the fourth week of the study, only 7 patients remained in each group (CCK+ and $\mathrm{CCK}-$ ) after the fourth week, which made the attainment of significance more difficult. It has been shown that higher septic rates can contribute to a worsening of PNAC and could have contributed to the higher bilirubin levels in the control group. ${ }^{15}$ Septic events, however, were identical in both groups, making this possibility unlikely. Although our current study was not randomized, we carefully selected controls that were matched to our study patients in terms of the greatest potential risk factors for the development of PNAC (ie, gestational age, diagnosis, and duration of TPN).

Studies in young rats receiving TPN have demonstrated that cholecystokinin is beneficial in reducing the degree of cholestasis..$^{16}$ In a more recent study by Curran et $\mathrm{al}^{17}$ on the use of Sincalide (CCK) in prepubescent rabbits maintained on TPN, the authors demonstrated that there was a reduction in periportal inflammation and fibrosis but no change in the balloon degeneration of the hepatocytes. This group also noted a significant increase in basal bile flow rates and clearance of sulfobromophthalein in the CCK-treated rabbits. Based on these reports, Rintala et $\mathrm{al}^{7}$ and our own group ${ }^{8}$ have successfully treated a number of premature and postsurgical neonates who developed significant degrees of PNAC. In each of these studies, several neonates attained a significant decline in their direct bilirubin levels after receiving IV cholecystokinin for 4 to 14 days. In our own study, several infants failed to respond to CCK; all were shown to have advanced PNAC with cirrhosis and subsequently were shown to have liver failure. The purpose of the current study was to evaluate the use of CCK in a prophylactic fashion to prevent the development of PNAC and, thus, cirrhosis.

Other tests of liver function failed to show any clear trends with CCK treatment. However, no study has shown that PNAC correlates well with any of these other reported tests.

Several adverse reactions may occur with the administration of cholecystokinin; these include gastrointestinal cramping and feeding intolerance because of spasm of the pyloric sphincter. No neonate developed any substantiated side effect during treatment with the CCK-octapeptide. Most likely this was due to the fairly low doses of the drug used in this study compared with our previous investigation in which doses several times higher were used to treat clinically advanced PNAC. ${ }^{8}$

In conclusion, use of cholecystokinin-octapeptide appears to be associated with a trend toward lower direct bilirubin levels in neonates receiving long-term TPN. Based on this finding, we believe that cholecystokinin-octapeptide may be effective in preventing the development of, or a reduction in the degree of parenteral nutrition-associated cholestasis in neonates. Future, randomized prospective studies should confirm the findings presented here. 


\section{REFERENCES}

1. Merritt R: Cholestasis associated with total parenteral nutrition. $J$ Pediatr Gastroenterol Nutr 5:9-22, 1986

2. Drongowski RA, Coran AG: An analysis of factors contributing to the development of total parenteral nutrition-induced cholestasis. JPEN 13:586-589, 1989

3. Bell R, Ferry G, Smith E: Total parenteral nutrition-associated cholestasis in infants. JPEN 10:356-359, 1986

4. Magnusson I, Thulin L, Einarsson K: Effects of substance P and somatostatin on taurocholate-stabilized and CCK- or secretin-induced choleresis in the anesthetized dog. Scand J Gastroenterol 19:10071014,1984

5. Magnusson I, Thulin L: Effect of substance P on CCK- or VIP-induced choleresis in anesthetized dogs. Acta Physiol Scand 106:293-297, 1979

6. Kaminski DL, Rose RC, Nahrwold DL: Effect of pentagastrin on cholecystokinin and secretin choleresis in the dog. J Surg Res 17:26-29, 1974

7. Rintala RJ, Lindahl H, Pohjavuori M: Total parenteral nutrition-associated cholestasis in surgical neonates may be reversed by intravenous cholecystokinin: A preliminary report. J Pediatr Surg 30:827830,1995

8. Teitelbaum DH, Han-Markey T, Schumacher RE: Treatment of parenteral nutrition-associated cholestasis with cholecystokinin-octapeptide. J Pediatr Surg 30:1082-1085, 1995

9. Sandler R, Kleinman R: IN Pediatric Gastrointestinal Disease, Walker
W, Durie P, Hamilton J, et al (eds). BC Decker, Inc, Philadelphia, 1991, pp 1065-1071

10. Teitelbaum D, Drongowski R, Spivak D: Rapid development of hyperbilirubinemia in infants with the short bowel syndrome as a correlate to mortality: Possible indication for early small bowel transplantation. Transplant Proc 28:2677-2678, 1996

11. Ginn-Pease M, Pantalos D, King D: TPN-associated hyperbilirubinemia: A common problem in newborn surgical patients. J Pediatr Surg 20:436-439, 1985

12. Touloukian $\mathrm{R}$, Seashore J: Hepatic secretory obstruction with total parenteral nutrition in the infant. J Pediatr Surg 10:353-360, 1975

13. Sitzman J, Pitt H, Steinborn P, et al: Cholecystokinin prevents parenteral nutrition induced biliary sludge in humans. Surg Gynecol Obstet 170:25-31, 1990

14. Teitelbaum D, O'Dorisio T, Gaginella T, et al: Effects of Caerulein on rat small and large intestinal fluid transport, in vivo. Reg Peptide 4:97105,1982

15. Beath S, Davies P, Papadpoulou A, et al: Parenteral nutrition-related cholestasis in postsurgical neonates: Multivariate analysis of risk factors. J Pediatr Surg 31:604-606, 1996

16. Innis S: Effect of cholecystokinin-octapeptide on total parenteral nutrition-induced changes in hepatic bile secretion and composition in the rat. J Pediatr Gastroenterol Nutr 5:793-798, 1986

17. Curran TJ, Uzoaru I, Das JB, et al: The effect of cholecystokinin-octapeptide on the hepatobiliary dysfunction caused by total parenteral nutrition. J Pediatr Surg 30:242-246, 1995 